\title{
Investigation of angiogenetic pathways in nasal polyposis
}

\author{
ALEXANDROS D. KARATZANIS ${ }^{2,3^{*}}$, KATERINA D. SAMARA ${ }^{1,2^{*}}$, KATERINA M. ANTONIOU ${ }^{1,2}$, \\ RENA LYMBOURIDOU ${ }^{2}$, NIKOLAOS CHATZAKIS ${ }^{3}$, DEMETRIOS A. SPANDIDOS ${ }^{4}$, \\ GEORGIOS A. VELEGRAKIS ${ }^{3}$ and NIKOLAOS M. SIAFAKAS ${ }^{1,2}$ \\ ${ }^{1}$ Department of Thoracic Medicine, University Hospital of Heraklion; ${ }^{2}$ Laboratory of Molecular \\ and Cellular Pulmonary Medicine; ${ }^{3}$ Department of Otolaryngology; ${ }^{4}$ Laboratory of Virology, \\ Medical School, University of Crete, Heraklion, Crete, Greece
}

Received February 10, 2012; Accepted February 16, 2012

DOI: $10.3892 / \mathrm{mmr} .2012 .819$

\begin{abstract}
Tissue angiogenesis is a complex phenomenon that results in the growth of new blood vessels from the microcirculation. This process has been known to play a crucial role in tumor growth as well as several benign diseases. The aim of this study was to assess mRNA expression of various angiogenic factors and chemokines in nasal polyps and compare the results to normal nasal mucosa. mRNA expression was measured using real-time RT-PCR for the following angiogenic factors and chemokines: VEGF, VEGFR-1, Ang-1, Ang-2, Tie-2A, Tie-2B, SDF-1 $\alpha$, SDF-1 $\beta$, CXCR4 and YY1. Biopsy specimens from nasal polyps in the polyposis group and middle turbinates in the control group were studied. A total of 18 nasal polyposis patients were studied and compared to 10 control subjects. Results showed VEGF, VEGFR-1, Ang-1, Ang-2, Tie-2A, Tie-2B, SDF- $1 \alpha$ and SDF-1 $\beta$ mRNA expression to be significantly higher in nasal polyposis patients compared to the control group $(p<0.05)$. The findings of this study support the role of angiogenic growth factors in the pathogenenesis of nasal polyposis. Further studies are required to confirm these results and evaluate potential clinical implications.
\end{abstract}

\section{Introduction}

Nasal polyposis (NP) constitutes a chronic inflammatory disease of the nose and sinuses characterized by the appearance of protruding tissue swellings of the nasal mucosa. These swellings most commonly originate from the middle meatus and anterior ethmoids. On histologic examination, they are

Correspondence to: Dr Katerina M. Antoniou, Department of Thoracic Medicine, University Hospital, Medical School, University of Crete, Heraklion 71110, Crete, Greece

E-mail: katerinaantoniou@yahoo.gr

*Contributed equally

Key words: angiogenesis, nasal polyps, vascular endothelial growth factor, angiopoietin, stromal-cell derived factor 1, middle turbinate typically characterized by proliferation of the epithelial layer, glandular hyperplasia, thickening of the basal membrane, focal fibrosis, edema and cellular infiltration of the stromal layer. Nasal polyps are relatively common in the general population and significantly affect patient quality of life. Despite these facts, etiology of the disease remains largely unknown (1-4).

Tissue angiogenesis is a complex phenomenon that results in the growth of new blood vessels from the microcirculation (5-7). This process has been known to play a crucial role in tumor growth, where it is regarded as a prerequisite allowing for the expansion of tumor populations beyond the size restricted by oxygen and nutrient diffusion (6). It also facilitates tumor progression and metastasis by causing degradation of the epithelial basement membrane and providing cancer cells with new capillaries for entering the circulation $(7,8)$. Increasing evidence shows that angiogenesis may also play a critical role in benign disorders $(9,10)$. The phenomenon, appearing in the form of growth of new blood vessels or the enlargement of existing ones, has been strongly associated with airway remodeling in chronic asthma and chronic obstructive pulmonary disease $(11,12)$. In addition, using immunohistochemistry it has been shown that vascular endothelial growth factor (VEGF), possibly the most important driver of angiogenesis, along with its receptors, are strongly expressed in nasal polyp tissues, thus supporting a role in the development of chronic inflammation and edema in this disease (13).

Real-time reverse transcription-polymerase chain reaction (RT-PCR) is a valuable method used for quantifying mRNA expression in biological samples. It is characterized by high sensitivity and accurate quantification output. To the best of our knowledge, there is a lack of studies evaluating the role of angiogenesis and angiogenic factors in NP using this method. The aim of the present study was to assess mRNA expression of various angiogenic factors and chemokines, including VEGF, Fms-related tyrosine kinase 1 (VEGF receptor- 1 or FLT-1), angiopoietin-1 (Ang-1), angiopoietin-2 (Ang-2), angiopoietin receptors Tie-2A and Tie-2B, stromal cell-derived factor $1 \alpha$ and $\beta$ (SDF- $1 \alpha$ and SDF-1 $\beta$ ), their receptor chemokine receptor-4 (CXCR4) and transcriptional repressor protein Yin-Yang-1 (YY1) in nasal polyps, and compare the results to normal nasal mucosa. 


\section{Patients and methods}

Patients. A total of 18 patients (11 males and 7 females) with NP undergoing endoscopic sinus surgery were studied. Patients were aged between 31 and 60 years, and had bilateral nasal polyps on endoscopy, with stage two or three, according to the Lildholdt clinical scoring system. In addition, all 18 patients were lifelong non-smokers, with a negative history of aspirin sensitivity. Furthermore, the patients were non-allergic according to a negative medical and family history, and negative RAST tests (C.A.R.L.A. ${ }^{\circledR}$, Capture Assay Radim Liquid Allergen; RADIM SpA, Rome, Italy; RAST) for common allergens, including dust mites (dermatophagoides pteronyssimus and farinae), cat and dog epithelia, olive, aspergillus fumingatus, alternaria alternata, parietaria judaica and ragweed.

A control group was also studied, comprising 10 adult individuals ( 7 males and 3 females) undergoing surgery for nasal septum deviation. All 10 cases had a negative history and clinical examination for nasal polyps. These individuals were lifelong non-smokers and also non-allergic, according to their medical and family history as well as RAST tests for all the allergens mentioned above.

No subjects with a history of local or systemic steroid use within the last 4 weeks prior to examination were included in the study.

Biopsy specimens from nasal polyps in the polyposis group and middle turbinates in the control group were obtained during surgery.

RNA isolation and RT-PCR. Total RNA was extracted from each sample using a power homogenizer and the TRIzol ${ }^{\circledR}$ reagent (Invitrogen, Carlsbad, CA, USA) according to the manufacturer's instructions. cDNA was synthesized using the Strascript reverse transcriptase kit (Stratagene, La Jolla, CA, USA) as previously described (14).

Real-time RT-PCR. RNA expression was measured using a real-time RT-PCR assay with SYBR-Green I. Primers were designed to span introns. $\beta$-actin was used as the internal control to normalize VEGF, FLT-1, Ang-1, Ang-2, Tie-2A, Tie-2B, SDF-1 $\alpha$, SDF-1 $\beta$, CXCR4 and YY1 (15-19) (Table I). In particular, $1 \mu \mathrm{l}$ cDNA from all patient and control samples was amplified in a PCR reaction containing $2 \mathrm{X}$ Brilliant SYBR-Green I QPCR Master Mix, $300 \mathrm{nM}$ of each primer and $30 \mu \mathrm{M}$ ROX passive reference dye, in a final volume of $20 \mu \mathrm{l}$. Following an initial denaturation at $95^{\circ} \mathrm{C}$ for $10 \mathrm{~min}$, the samples were subjected to 40 cycles of amplification, comprising denaturation at $95^{\circ} \mathrm{C}$ for $30 \mathrm{sec}$, annealing at appropriate temperatures for each primer pair for $30 \mathrm{sec}$ and elongation at $72^{\circ} \mathrm{C}$ for $30 \mathrm{sec}$, followed by a melt curve analysis, in which the temperature was increased from 55 to $95^{\circ} \mathrm{C}$ at a linear rate of $0.2^{\circ} \mathrm{C} / \mathrm{sec}$. Data collection was performed during annealing and extension, with two measurements at each step, and at all times during the melt curve analysis. In each PCR reaction, two non-template controls were included. The PCR experiments were conducted on the Mx3000P real-time PCR thermal cycler using the software version 2.00 (Stratagene).

To verify the results of the melt curve analysis, PCR products were analyzed by electrophoresis in $2 \%$ agarose gels, stained with ethidium bromide and photographed on a UV light transilluminator. Primer sequences were used, and

Table I.Primer sequences used for quantitative real-time RT-PCR.

Gene

Primer pair sequence $\left(5^{\prime}-3^{\prime}\right)$

Annealing temperature $\left({ }^{\circ} \mathrm{C}\right)$

VEGF F: ATGACGAGGGCCTGGAGTGTG R: CCTATGTGCTGGCCTTGGTGAG

FLT-1 F: CGGCGGCGGCGAACGAG R: CATGATGTGCTGGGTGCCTTTTA

CXCR4 F: GGTGGTCTATGTTGGCGTCT R: TGGAGTGTGACAGCTTGGAG

Ang-1 F: TTAATGGACTGGGAAGGGAACC R: AGGGGCCACAAGCATCAAA

Ang-2 F: AGCCGGCAAAATAAGCAGCATC R: GGTTGTGGCCTTGAGCGAATAGC

Tie-2A F: GGGCATCCTGGACCTGTGAGAC R: CCCCTGGGCCTTGGTGTTGACT

Tie-2B F: AATGAGACAATGCTGGCGGGAGAA R: ATGCCAGTGAAAGGGAAACAGAGG

SDF-1 $\alpha$ F: TGAGAGCTCGCTTTGAGTGA R: CACCAGGACCTTCTGTGGAT

SDF-1 $\beta$ F: CTAGTCAAGTGCGTCCACGA R: GGACACACCACAGCACAAAC

YY1 F: GGAATACCTGGCATTGACC R: TCTTTGTGCAGCCTTTGAG

$\beta$-actin F: CGGCATCGTCACCAACTG R: GGCACACGCAGCTCATTG

annealing temperatures and PCR product length for all the markers as well as for $\beta$-actin were analyzed (Table I). The reactions were run in triplicate and transcript levels were calculated and normalized to each specimen's housekeeping gene mRNA ( $\beta$-actin), as well as the appropriate calibrators, using the $\Delta \Delta C t$ method for relative quantification (12). After amplification, standard curves were constructed from samples used in a series of consecutive dilutions, for both the gene of interest and the internal control ( $\beta$-actin). The markers and $\beta$-actin amplification efficiencies were identical, reaching $100 \%$. Data from the two patient groups were first normalized against variation in sample quality and quantity. Normalized values to $\beta$-actin, $\Delta \mathrm{Cts}$, were initially calculated using the equation: $\Delta \mathrm{Ct}_{\text {sample }}=\mathrm{Ct}_{\mathrm{TLRs}}-\mathrm{Ct}_{\beta \text {-actin }}$.

Statistical analysis. VEGF, FLT-1, Ang-1, Ang-2, Tie-2A, Tie-2B, SDF-1 $\alpha$, SDF-1 $\beta$, CXCR4 and YY1 mRNA levels were first evaluated by the one-sample Kolmogorov-Smirnov goodness of fit test to determine whether they exhibited a normal distribution or no distribution. Values reported are the means \pm standard error of the mean (SEM). The nonparametric Mann-Whitney U test was used for post hoc comparisons. The Chi-square test was used, as indicated, to examine the VEGF, VEGFR-1, Ang-1, Ang-2, Tie-2A, Tie-2B, SDF-1 $\alpha$, SDF-1 $\beta$, CXCR4 and YY1 expression status among the nasal polyps group and controls. Statistical analysis was carried out using SPSS 17.0 (SPSS Inc., Chicago, IL, USA). Statistical significance was set at the $95 \%$ level $(\mathrm{p}<0.05)$. 
Table II. mRNA expression of markers studied in nasal polyposis (NP) patients and control subjects.

\begin{tabular}{lccc}
\hline Markers & NP & Controls & p-value \\
\hline FLT-1 & $647.80 \pm 378.5$ & $320.90 \pm 320.80$ & 0.040 \\
Ang-1 & $331.47 \pm 318.2$ & $0.048 \pm 0.023$ & 0.009 \\
Ang-2 & $1,432.50 \pm 1,283.7$ & $13.10 \pm 12.80$ & 0.050 \\
Tie-2A & $282.70 \pm 253.8$ & $6.90 \pm 6.80$ & 0.010 \\
Tie-2B & $62.30 \pm 36.4$ & $9.39 \pm 9.33$ & 0.005 \\
VEGF & $444.10 \pm 443.1$ & $0.57 \pm 0.12$ & 0.040 \\
CXCR4 & $667.70 \pm 661.4$ & $32.30 \pm 13.30$ & NS \\
SDF-1 $\alpha$ & $28.50 \pm 11.3$ & $4.00 \pm 2.90$ & 0.001 \\
SDF-1 $\beta$ & $44.80 \pm 16.8$ & $4.20 \pm 3.50$ & 0.020 \\
YY1 & $366.90 \pm 360.1$ & $13.90 \pm 13.50$ & NS
\end{tabular}

Values are shown as the means \pm SEM. p $<0.05$ was considered statistically significant. NS, non significant.

\section{Results}

Demographics. Patients in the two groups were of comparable age. The NP patients (11 males and 7 females) had a median age of $42.3 \pm 1.6$ years. The control group ( 7 males and 3 females) had a median age of $38.4 \pm 3.5$ years. Patients in both groups were lifelong non-smokers and non-allergic.

$m R N A$ expression. Table II shows the mRNA values for the markers studied. VEGF, FLT-1, Ang-1, Ang-2, Tie-2A, Tie-2B, SDF- $1 \alpha$ and SDF- $1 \beta$ mRNA expression was found to be significantly higher in NP patients compared to the control group $(\mathrm{p}<0.05)$. Ang-2, Tie-2A and Tie-2B mRNA expression $(\%)$ was found to be significantly higher in the control subjects compared to the NP patients $(\mathrm{p}<0.05)$ (Table III). No statistically significant difference was detected at the mRNA expression levels of CXCR4 and YY1 for either of the two groups studied.

\section{Discussion}

In the present study, we evaluated the role of angiogenic factors and other chemokines in nasal polyposis. The hypothesis that such a role exists was based on the numerous similarities present with regard to remodeling patterns and histopathologic features between bronchial asthma and nasal polyposis. Our main finding was the increased expression of mRNA levels of VEGF, FLT-1, Ang-1, Ang-2, Tie-2A, Tie-2B, SDF- $1 \alpha$ and $\mathrm{SDF}-1 \beta$ in NP tissue samples compared to normal turbinate tissue samples. There is conflicting evidence regarding the exact role of angiogenic growth factors in the pathophysiology of nasal polyposis (20-24). It has been suggested that proangiogenic factors are involved in the disease process through the formation of edema, the activation of inflammatory cell migration and activation of the nitric oxide synthase pathway $(13,20-22)$.

The etiology of nasal polyps remains unknown. Several theories have been postulated to explain the pathogenesis of nasal polyps, although none of these theories have adopted
Table III. Percentage of subjects expressing mRNA in nasal polyposis (NP) patients and control subjects.

\begin{tabular}{lrcc}
\hline Markers & NP $(\%)$ & Controls $(\%)$ & p-value \\
\hline FLT-1 & $8 / 18$ & $6 / 10$ & NS \\
Ang-1 & $11 / 18$ & $7 / 10$ & NS \\
Ang-2 & $9 / 18$ & $7 / 10$ & 0.04 \\
Tie-2A & $6 / 18$ & $7 / 10$ & $<10^{-4}$ \\
Tie-2B & $6 / 18$ & $7 / 10$ & $<10^{-4}$ \\
VEGF & $14 / 18$ & $8 / 10$ & NS \\
CXCR4 & $16 / 18$ & $9 / 10$ & NS \\
SDF-1 $\alpha$ & $17 / 18$ & $10 / 10$ & NS \\
SDF-1 $\beta$ & $17 / 18$ & $10 / 10$ & NS \\
YY1 & $6 / 18$ & $4 / 10$ & NS \\
\hline
\end{tabular}

Chi-square test was used. $\mathrm{p}<0.05$ was considered statistically significant. NS, non significant.

all available data $(2,4,25-27)$. Several investigators believe that polyps are an exvagination of the normal nasal or sinus mucosa that is filled with edematous stroma. Other investigators believe polyps are a distinct entity arising from the mucosa (2,27). A number of potential alterations in the respiratory epithelium occur after insult and may eventually lead to the clinical picture of polyposis (27). Genetic susceptibility may be a prerequisite, at least in certain cases of polyposis (4). Regardless, however, of the exact molecular pathway, most theories consider polyps to be the ultimate manifestation of chronic inflammation (25-27). Therefore, conditions leading to chronic inflammation in the nasal cavity lead to nasal polyps. The condition most commonly associated with multiple benign nasal polyps is bronchial asthma, which is present in $20-50 \%$ of cases with nasal polyposis (2).

VEGF is considered the most potent pro-angiogenic mediator in humans. It is a potent inducer of endothelial proliferation, migration and survival, while it also acts as a pro-inflammatory cytokine $(28,29)$. The present study provides evidence supporting an important role for VEGF in the pathogenesis of nasal polyposis, as we measured significantly high levels of mRNA expression both for VEGF and its receptor FLT-1 in nasal polyps compared to control turbinate mucosa. These findings are in accordance with recent literature (20-24), although, to the best of our knowledge, this is the first study to report mRNA expression levels of both VEGF and its receptor FLT-1 in human nasal polyp tissue. Mounting evidence suggests that, besides its influence on blood vessel formation, VEGF may even be directly involved in the pathogenesis of bronchial asthma $(30,31)$. Similarly, we hypothesized that VEGF may mediate upper airway remodeling observed in the development of sinonasal polyposis. VEGF has been found to contribute to non-specific airway hyper-responsiveness, exert chemotactic effects on eosinophils and enhance smooth muscle cell proliferation. Th2 cells, a key element in bronchial asthma pathophysiology, have been shown to express receptors for VEGF. Moreover, evidence exists showing that high VEGF levels may be directly associated with asthma severity, as 
they correlate directly with disease activity and inversely with airway caliber and airway responsiveness $(30,31)$. VEGF has been shown not only to promote cell growth, but also to inhibit apoptosis in epithelial cells and to auto-regulate its own production in a positive manner in non-neoplastic primary human airway epithelial cells. Thus, VEGF promotes the development of epithelial cell hyperplasia, one of the key features observed in nasal polyposis.

Angiopoietins are a family of angiogenic factors, Ang-1, -2, -3 and -4 (29). These factors are thought to be major mediators of angiogenesis and microvascular remodeling $(29,32)$. Angiopoietins function by binding their physiologic receptors, Tie-1 and Tie-2. These are receptor tyrosine kinases, so termed because they mediate cell signals by inducing the phosphorylation of key tyrosines, thus initiating cell signaling. In our study, Ang-1, Ang-2, Tie-2A and Tie-2B mRNA expression levels were found to be significantly higher compared to the controls. Little evidence exists regarding these genes in nasal polyposis, where an imbalance between Ang-1 and Ang-2 was found using enzyme-linked immunosorbent assay (ELISA) (21). Tie receptor levels were not measured before and, to the best of our knowledge, this is the first time these receptor levels are evaluated in nasal polyposis. Although it remains unclear which receptor mediates functional signals, evidence shows that Tie-2 is capable of physiologic activation as a result of binding the angiopoietins $(5,12)$. Ang- 1 and Ang- 2 compete for binding sites on the Tie-2 receptor with similar affinity. Ang-2 is an antagonist and does not induce the phosphorylation of Tie- 2 receptors. Ang-2 is usually expressed at remodeling sites in the adult where it inhibits the stabilizing effect of Ang-1. Ang-1 interacts with VEGF in a coordinated manner in the formation of blood vessels during angiogenesis, although Ang-1 may induce angiogenesis even in the absence of VEGF (12). In a recent study on severe asthma, the two angiopoietins levels were higher compared to the healthy subjects. In severe asthma, Ang-2 is associated with mediators involved in both the inflammatory and the vascular remodeling processes (31). Ang-1, however, has been shown to induce pro-inflammatory effects, such as eosinophil chemotaxis, and also contributes to the increased secretion of matrix metalloproteinase- 2 as well as the decreased secretion of tissue inhibitors of metalloproteinase-2 (33). Increased activity of proteolytic enzymes, including metalloproteinases, has been suggested to be involved in airway remodeling.

Stromal-derived factor 1 (SDF-1, CXCL12) is a CXC chemokine that exhibits angiogenic activity, and its receptor is CXCR4 (34). SDF-1 is cleaved into two chains, namely SDF-1 $\alpha$ and SDF-1 $\beta$, which show a reduced chemotactic activity. In this study, SDF-1 $\alpha$ mRNA levels were significantly increased in polyp tissue compared to turbinate tissue from controls. A recent study, which evaluated the presence of B-cell attracting chemokines in the pathogenesis of chronic rhinosinusitis with nasal polyps using ELISA, also reported elevated SDF- $1 \alpha$ protein levels in polyp tissue compared to turbinate tissue (35). It has been demonstrated that there is a positive feedback loop of VEGF-inducing CXCR4 and SDF-1 expression by endothelial cells and conversely SDF-1 interaction with CXCR4 enhances VEGF expression of these cells (18). SDF-1 may account for an increased presence of B-cells and their products in chronic sinusits, contributing to eosinophilic inflammation in patients with nasal polyps (35).
YY1 is a multifunctional transcription factor that exhibits positive and negative control on a large number of cellular genes, and may be important in their development and differentiation (36). YY1 binds to the promoter regions of several T-cell cytokine genes, but the expression and contribution of this factor to cytokine gene expression and T-cell activation in vivo is not clear. Previous reports have indicated that YY1 regulates T-cell cytokine gene expression and allergic immune responses in a gene dose-dependent manner (37). Moreover, previous studies have shown that altered YY1 binding due to the TGF- $\beta 1$ polymorphism may modulate airway inflammation and remodeling, thereby increasing susceptibility to bronchial asthma (38). As expected, higher levels of YY1 were found in nasal polyps compared to normal mucosa in this study, although statistical significance was not achieved.

In conclusion, the study of the molecular basis of a disease is of fundamental importance as it offers better insight into the pathogenesis and opens new, disease-specific therapeutic trends. This study utilized real-time RT-PCR to assess the mRNA levels of angiogenic growth factors in nasal polyp tissues. Most factors assessed along with their respective receptors, VEGF, VEGFR-1, Ang-1, Ang-2, Tie-2A, Tie-2B, SDF- $1 \alpha$ and SDF-1 $\beta$, were found to be significantly increased in nasal polyps compared to normal middle turbinate mucosa. These findings support the role of angiogenic growth factors in the pathogenenesis of nasal polyposis. However, further studies are required to confirm these results and evaluate potential clinical implications.

\section{References}

1. Andrews AE, Bryson JM and Rowe-Jones JM: Site of origin of nasal polyps: relevance to pathogenesis and management. Rhinology 43: 180-184, 2005.

2. Fokkens WJ, Lund V, Bachert C, et al: European position paper on rhinosinusitis and nasal polyps. Rhinol Suppl 18: 1-87, 2005.

3. Rinia AB, Kostamo K, Ebbens FA, van Drumen CM and Fokkens WJ: Nasal polyposis: a cellular based approach to answering questions. Allergy 62: 348-358, 2007.

4. Karatzanis AD, Tzortzaki E, Samara KD, et al: Microsatellite DNA instability in nasal polyposis. Laryngoscope 119: 751-756, 2009.

5. De Paulis A, Prevete N, Fiorentino I, et al: Expression and functions of the vascular endothelial growth factors and their receptors in human basophils. J Immunol 177: 7322-7331, 2006.

6. Carmeliet P and Jain RK: Angiogenesis in cancer and other diseases. Nature 407: 249-257, 2000.

7. Rodrigo JP, Suarez C, Ferlito A, et al: Potential molecular prognostic markers for lymph node metastasis in head and neck squamous cell carcinoma. Acta Otolaryngol 123: 100-105, 2003.

8. Economidou F, Margaritopoulos G, Antoniou KM and Siafakas NM: The angiogenetic pathway in malignant pleural effusions: Pathogenetic and therapeutic implications. Exp Ther Med 1: 3-7, 2010.

9. Hoshino M, Nakamura Y and Hamid QA: Gene expression of vascular endothelial factor and its receptors and angiogenesis in bronchial asthma. J Allergy Clin Immunol 107: 1034-1038, 2001.

10. Ikeda M, Hosoda Y, Hirose S, Okada Y and Ikeda E: Expression of vascular endothelial grow th factor isoforms and their receptors Flt-1, KDR, and neuropilin-1 in synovial tissues of rheumatoid arthritis. J Pathol 191: 426-433, 2000.

11. Siafakas NM, Antoniou KM and Tzortzaki EG: Role of angiogenesis and vascular remodelling in chronic obstructive pulmonary disease. Int J Chron Obstruct Pulmon Dis 2: 453-462, 2007.

12. Makinde T, Murphy RF and Agrawal DK: Immunomodulatory role of vascular endothelial growth factor and angiopoietin-1 in airway remodeling. Curr Mol Med 6: 831-841, 2006. 
13. Wittekindt C, Hess A, Bloch W, Sultanie S and Michel O: Immunohistochemical expression of VEGF and VEGF receptors in nasal polyps as compared to normal turbinate mucosa. Eur Arch Otorhinolaryngol 259: 294-298, 2002.

14. Margaritopoulos GA, Antoniou KM, Karagiannis K, et al: Investigation of Toll-like receptors in the pathogenesis of fibrotic and granulomatous disorders: a bronchoalveolar lavage study. Fibrogenesis Tissue Repair 3: 20, 2010.

15. Antoniou KM, Soufla G, Proklou A, et al: Different activity of the biological axis VEGF-Flt-1 (fms-like tyrosine kinase 1) and CXC chemokines between pulmonary sarcoidosis and idiopathic pulmonary fibrosis: a bronchoalveolar lavage study. Clin Dev Immunol: 537929, 2009.

16. Antoniou KM, Soufla G, Lymbouridou R, et al: Expression analysis of angiogenic growth factors and biological axis CXCL12/CXCR4 axis in idiopathic pulmonary fibrosis. Connect Tissue Res 51: 71-80, 2010.

17. Margaritopoulos GA, Antoniou KM, Karagiannis K, et al: Investigation of angiogenetic axis Angiopoietin-1 and -2/Tie-2 in fibrotic lung diseases: a bronchoalveolar lavage study. Int J Mol Med 26: 919-923, 2010.

18. Margaritopoulos GA, Antoniou KM, Soufla G, et al: Upregulation of stromal cell derived factor-1alpha in collagen vascular diseases-associated interstitial pneumonias (CVDs-IPs). Pulm Pharmacol Ther 23: 115-120, 2010.

19. Margaritopoulos GA, Antoniou KM, Soufla G, Vassalou E, Spandidos DA and Siafakas NM: Yin Yang-1 (YY-1) expression in idiopathic pulmonary fibrosis. J Recept Signal Transduct Res 31: 188-191, 2011.

20. Lee HS, Myers A and Kim J: Vascular endothelial growth factor drives autocrine epithelial cell proliferation and survival in chronic rhinosinusitis with nasal polyposis. Am J Respir Crit Care Med 180: 1056-1067, 2009.

21. Park SK, Kim HI and Yang YI: Roles of vascular endothelial growth factor, Angiopoietin 1, and Angiopoietin 2 in nasal polyp. Laryngoscope 119: 409-413, 2009.

22. Hirshoren N, Neuman T, Gross M and Eliashar R: Angiogenesis in chronic rhinosinusitis with nasal polyps and in antrochoanal polyps. Inflamm Res 60: 321-327, 2011.

23. Ahmed SK, Williams JL, Drake-Lee A and Egginton S: No significant role for angiogenesis in nasal polyposis. Am J Rhinol 22: 24-28, 2008.

24. Zaravinos A, Soufla G, Bizakis J and Spandidos DA: Expression analysis of VEGFA, FGF2, TGF- $\beta 1$, EGF and IGF1 in human nasal polyposis. Oncol Rep 19: 385-391, 2008.
25. Bernstein JM, Gorfien $\mathbf{J}$ and Noble B: Role of allergy in nasal polyposis: a review. Otolaryngol Head Neck Surg 113: 724-732, 1995.

26. Andrews AE, Bryson JM and Rowe-Jones JM: Site of origin of nasal polyps: relevance to pathogenesis and management. Rhinology 4: 180-184, 2005.

27. Bernstein JM: Update on the molecular biology of nasal polyposis. Otolaryngol Clin North Am 38: 1243-1255, 2005.

28. McDonald DM: Angiogenesis and remodeling of airway vasculature in chronic inflammation. Am J Respir Crit Care Med 164: 39-45, 2001.

29. Thurnston G, Murphy TJ, Baluk P,Lindsey JR and McDonald DM: Angiogenesis in mice with chronic airway inflammation: straindependent differences. Am J Pathol 153: 1099-1112, 1998.

30. Asai K, Kanazawa H, Kamoi H, Shiraishi S, Hirata K and Yoshikawa J: Increased levels of vascular endothelial growth factor in induced sputum in asthmatic patients. Clin Exp Allergy 33: 595-599, 2003.

31. Tseliou E, Bakakos P, Kostikas K, et al: Increased levels of angiopoietins 1 and 2 in sputum supernatant in severe refractory asthma. Allergy 67: 396-402, 2012.

32. Oike Y, Ito Y, Maekawa $\mathrm{H}$, et al: Angiopoietin-related growth factor (AGF) promotes angiogenesis. Blood 103: 3760-3765, 2004.

33. Cannady SB, Batra PS, Leahy R, et al: Signal transduction and oxidative processes in sinonasal polyposis. J Allergy Clin Immunol 120: 1346-1353, 2007.

34. Shirozu M, Nakano T, Inazawa J, et al: Structure and chromosomal localization of the human stromal cell-derived factor 1 (SDF1) gene. Genomics 28: 495-500, 1995.

35. Patadia M, Dixon J, Conley D, et al: Evaluation of the presence of B-cell attractant chemokines in chronic rhinosinusitis. Am J Rhinol Allergy 24: 11-16, 2010.

36. Cai Y, Jin J, Yao T, et al: YY1 functions with INO80 to activate transcription. Nat Struct Mol Biol 14: 872-874, 2007.

37. Guo J, Lin X, Williams MA, Hamid Q and Georas SN: Yin-Yang regulates effector cytokine gene expression and $\mathrm{T}(\mathrm{H}) 2$ immune responses. J Allergy Clin Immunol 122: 195-201, 2008.

38. Silverman ES, Palmer LJ, Subramaniam V, et al: Transforming growth factor-betal promoter polymorphism C-509T is associated with asthma. Am J Respir Crit Care Med 169: 214-219, 2004. 